\title{
Peter Christian Jacobsen (éd. et trad.), Die Geschichte vom Leben des Johannes, Abt des Klosters Gorze
}

\section{Tristan Martine}

\section{OpenEdition}

\section{Journals}

Édition électronique

URL : http://journals.openedition.org/ifha/8634

DOI : $10.4000 /$ ifha.8634

ISSN : 2198-8943

Éditeur

IFRA - Institut franco-allemand (sciences historiques et sociales)

Référence électronique

Tristan Martine, «Peter Christian Jacobsen (éd. et trad.), Die Geschichte vom Leben des Johannes, Abt des Klosters Gorze », Revue de l'IFHA [En ligne], Date de recension, mis en ligne le 01 février 2017, consulté le 24 septembre 2020. URL : http://journals.openedition.org/ifha/8634 ; DOI : https://doi.org/ $10.4000 /$ ifha.8634

Ce document a été généré automatiquement le 24 septembre 2020.

(C)IFHA 


\section{Peter Christian Jacobsen (éd. et trad.), Die Geschichte vom Leben des Johannes, Abt des Klosters Gorze}

Tristan Martine

\section{RÉFÉRENCE}

Peter Christian Jacobsen (éd. et trad.), Die Geschichte vom Leben des Johannes, Abt des Klosters Gorze, Wiesbaden: Harrasowitz Verlag (Monumenta Germaniae Historica. Scriptores Rerum Germanicarum in Usum Scholarum Separatim Editi, LXXXI), 2016, IX +629 p., $80 €$ 
En 2009, Peter Christian Jacobsen éditait et traduisait les «Miracles» écrits en l'honneur de saint Gorgon (ainsi que d'autres textes complémentaires), qui constituent une source importante pour comprendre la réforme de Gorze, effectuée vers 934 sous l'impulsion de l'évêque Adalbéron ${ }^{\mathrm{er}}$ de Metz (Miracula s. Gorgonii. Studien und Texte zur GorgoniusVerehrung im 10. Jahrhundert, 2009). Son volume fut disponible quelques semaines avant la parution, en France, d'une autre édition de ces mêmes Miracula, traduits en français (Monique Goullet, Michel Parisse, Anne Wagner, Sources hagiographiques de l'histoire de Gorze (X $X^{e}$ siècle), 2010).

Sept ans plus tard, P.-C. Jacobsen publie un travail similaire à partir d'une nouvelle source liée à la précédente, la Vita Iohannis abbatis Gorziensis, texte célèbre qui avait déjà été édité une première fois dans le cadre des Monumenta Germaniae Historica en 1841 par G. H. Pertz. À la suite d'un colloque consacré à l'abbaye de Gorze en 1988 (Michel Parisse, Otto Gerhard Oexle (dir.), L'abbaye de Gorze au $X^{e}$ siècle, 1993), M. Parisse, alors directeur de la Mission historique française de Göttingen, décida de se lancer dans une entreprise de traduction de la Vita, aidé par différents collègues allemands et français. Publiée en 1999 (Michel Parisse, Jean de SaintArnoul, 1999), sa traduction se fondait sur l'édition du début du XIX ${ }^{\mathrm{e}}$ siècle, même si P.C. Jacobsen, déjà chargé par les MGH d'en produire une nouvelle édition critique, lui avait communiqué ses observations sur plusieurs parties du manuscrit. Ce volume représente l'aboutissement de ce travail.

Cette Vita constitue une source très précieuse, d'autant plus que nous n'avons conservé que très peu de sources littéraires pour cette période, le $\mathrm{X}^{\mathrm{e}}$ siècle, et cet espace, la Lorraine. Elle est extrêmement utile pour comprendre les mouvements de ce que l'on appelle la "réforme lotharingienne » ou même "réforme gorzienne ", et livre de riches renseignements sur les structures politiques de la Lotharingie post-carolingienne, d'autant que ses informations peuvent être croisées avec celles livrées par les Miracula sancti Gorgonii. Cette Vita fut rédigée par Jean de Saint-Arnoul, un proche de Jean de Gorze, entre 974 et 984 . Le texte met tout d'abord en scène la jeunesse de Jean et sa conversion, puis dresse le portrait des moines de Gorze, avant de revenir sur la figure de Jean, sur son activité pour le monastère et sur sa ferveur, faisant la liste détaillée de toutes ses actions, et se concluant par le récit de son ambassade à Cordoue.

Une introduction de plus de cent pages offre de nombreux éléments de contextualisation, analysant ce que l'on sait de la vie de Jean de Gorze, le système des ambassades (en l'occurrence celle que mena Jean auprès du calife omeyyade), la formation intellectuelle à Gorze, et enfin la personnalité de l'auteur de cette Vita et son travail. Fort d'une connaissance fine de l'historiographie récente, l'auteur apporte de nouveaux arguments sur différents points problématiques, comme la date de la mort de 
Jean de Gorze, sur laquelle il est en désaccord avec M. Parisse (p. 35-38), ou le lien complexe entre la Vita et les Miracula sancti Gorgonii (p. 94).

Le manuscrit unique de la Vita (manuscrit latin 13766 de la Bibliothèque nationale de France), qui n'est pas un autographe mais une copie de la fin du $\mathrm{X}^{\mathrm{e}}$ siècle, présente des lacunes, les marges ayant souffert de l'humidité et la fin du manuscrit comprenant de nombreuses rognures. G. H. Pertz les avait comblées pour rendre compréhensibles certaines phrases. Dans son édition, M. Parisse reproduisait entre crochets les ajouts du $\mathrm{XIX}^{\mathrm{e}}$ siècle, car le travail de G. H. Pertz était de qualité. L'éditeur a ici décidé de reléguer les hypothèses de G.H.Pertz dans l'apparat critique, sauf quand ses choix lui paraissaient incontestables, comme quand il s'agit de définir le nombre d'années d'absence de Jean de Gorze (p.455), effacé dans le manuscrit mais que d'autres éléments du texte permettent de déterminer avec quasi-certitude. Sur l'ensemble du texte, cela ne change presque rien au sens, même si, à deux ou trois reprises, cela modifie sensiblement la compréhension de quelques points de détail. Ainsi, lors de son ambassade à Cordoue, Jean était accompagné d'un seul moine et, selon G. H. Pertz comblant une lacune, suivi par M. Parisse, de cinq chevaux. Or en étudiant d'autres passages du texte, et notamment un épisode où la cour de Cordoue reproche à Jean que ses iuniores saluent de la tête les femmes dans les rues, P.-C. Jacobsen montre qu'on ne peut pas suivre l'hypothèse du XIX ${ }^{e}$ siècle (p.424) et que l'on n'avait en réalité pas fourni à Jean cinq chevaux, mais bien cinq jeunes laïcs chargés de le protéger, ce qui change l'allure de la délégation.

Autant qu'il soit possible à un Français d'en juger, la traduction allemande est fluide, séquençant le texte latin en unités courtes afin de le rendre plus clair. L'apparat critique est un modèle du genre, d'une très grande précision, croisant les sources, faisant systématiquement le point sur les éditions précédentes, renvoyant à une large bibliographie, tandis que les index, qui couvrent plus de 150 pages, sont d'une grande utilité. Cette nouvelle édition ne sera donc pas réservée aux chercheurs allemands, mais trouvera sa place dans la bibliothèque de tous ceux, bien plus nombreux, qui s'intéressent aux réformes monastiques et, de manière plus générale, à la Lotharingie du Xe siècle.

\section{INDEX}

Index chronologique : Moyen Âge

Thèmes : Sources

\section{AUTEURS}

\section{TRISTAN MARTINE}

Université Paris-Est Marne-la-Vallée, ACP Université de Lorraine, HISCANT-MA 